\title{
METHODOLOGICAL FRAMEWORK FOR CAPABILITY ANALYSIS
}

\author{
Josef MELICHAR, Josef PROCHÁZKA \\ University of Defence, Brno, The Czech Republic \\ josef.melichar@unob.cz, josef.prochazka@unob.cz
}

\begin{abstract}
The article offers a methodological framework for systematic and rigorous capability analysis under uncertainty while applying basic principles of capability based planning. More specifically, it focuses on determination of capability requirements against multiple generic and specific set of force development scenarios, identification of capability mismatches, operational risk assessment steaming from capability gaps and capability prioritisation techniques and procedures. Methodological framework for capability analysis is one of the outputs of the Centre for Security and Military Strategic Studies of University of Defence in Brno institutional research project "STRATAL" - Strategic Alternatives for the Development of the Czech Armed Forces. The authors offer an innovative methodology for further discussion and best practices for development of sound defence planning process and its analytical support. Successful application of this framework depends on the quality of professional military judgement and proper use of relevant analytical techniques and procedures. In addition to that, the framework interlinks efforts in several functions of overarching defence management system, and that constitutes certain risks for generating expected outcomes e.g. insufficient coordination and harmonisation of effort, unclear responsibility for final outcomes just to name a few.
\end{abstract}

Keywords: capability analysis, capability mismatches, capability gaps, operational risks, prioritisation

\section{Introduction}

Capability analysis methodological framework (CAMF) as a tool for systematic and rigorous capability analysis under uncertainty is one of the outcomes of institutional research project Strategic Alternatives for the Development of the Czech Armed Forces (STRATAL) which has been conducted by the Centre for Security and Military Strategic Studies of University of Defence in Brno since 2016. The CAMF provides an effective analytical method, techniques and procedures for determination of capability requirements against multiple generic and specific set of force development scenarios, identification of capability mismatches, operational risk assessment steaming from capability gaps and capability prioritisation. Its relevance has already been proved in several instances both at national and multinational level e.g. in force development in Canada [1], Norway [2] or NATO Defence Planning Process [3].

This article's ambition is to offer sound methodological framework for strategists, defense planners and other stakeholders involved in force development and strategic level decision making and planning in the area of national defense provision. It's main objective is to assist decision makers and strategic planners in addressing uncertainty and complexity once it comes to the determination of capability requirements, identification of capability mismatches, assessment of operational risks associated 
with capability gaps and capability gaps prioritisation.

It also could help understanding the impact of ever evolving security and operating environment and armed forces' mission on the development of strategically relevant, operationally credible and tactically decisive military force in long-term time span.

Long-term time span deals with the configuration of Future Armed Forces in the horizon from 15 to 30 years ahead. This time span allows for prudent reengineering of existing and planned defense posture, command and force structure and capabilities while taking into consideration both opportunities and challenges steaming from external environment [1].

Based on the CAMF, decision makers and planners can design multifunctional forces possessing capabilities with more flexibility that can ensure the defence organization has the required agility to remain competitive in the future, even in case the future differs from envisaged futures. It will also help to anticipate changes and prepare the organization for dealing with future challenges and taking advantage of emerging opportunities.

Critical precondition for successful application of the CAMF is the existence of professional military judgement combining scientific rigor with sound experience. Force development is an area which traditionally requires combination of science and military art. It uses variety of inputs including analysis and advice from national and allied defence science community, civilian defence employees, academia and industry. The CAMF strives for objectivity by avoiding wishful thinking, assimilating bad news, discarding pleasing interpretations that cannot pass the elementary test $\mathrm{s}$ of evidence and logic. Ultimately, the integrity of the process depends on synthesis, rather than competition, of disparate inputs.

Furthermore, it also spans several functions within overarching defence sector management system e.g. policy, planning, programming, project execution and budgeting. This facts and interconnections put certain level of risk on the CAMF implementation and generation of expected outcomes e.g. insufficient coordination and harmonisation of effort, unclear responsibility for final outcomes are just a few of them.

\section{Establishing the Process}

The CAMF is built on process embracing 7 following steps: (1) assessment of future forces mission; (2) force development scenario design (both generic and specific); (3) capability needs definition; (4) capability mismatches determination; (5) operational risks assessment steaming from capability gaps; (6) prioritization of capability requirements; (7) force development options consideration.

\subsection{Assessment of Future Forces Mission}

The force development process is influenced by a series of stand-alone, yet inter-related documents. These documents encompass a wide span of topics including policy, strategic direction and studies. The cornerstone of the CAMF is the government's security and defence strategy, firm and realistic grasp of future security and operating environment, trends in technology evolution and strategic defence concept. This strategic direction enables to define the boundaries of what a military force is expected to be able to achieve in that future environments - plausible future missions.

For the Czech Armed Forces the following future missions are foreseen: (1) strategic assault - defence of the country's territorial integrity and sovereignty in high-intensity conflict in context of collective defence; (2) limited assault - collective defence of any given ally against conventional enemy with comparable capability; (3) crisis response operation as part of broader international crisis management (peace enforcement and peace keeping under NATO, EU or UN umbrella); (4) post-conflict stabilization and reconstruction operations; (5) support to capacity building; (6) rescue and 
humanitarian operations abroad; (7) operation in support to national crisis management or assistance to law enforcement agencies (terrorist attack, natural or technical disasters, migration, epidemic, major culture or sport event); (8) air defence, and (9) cyber defence [4].

\subsection{Force Development Scenario Design}

Force Development Scenario (FDS) serves specifically for capability analysis in order to define capability needs as well as verify capability of existing force and command structures. It works backwords from what you want to achieve (political and military end states, strategic objectives and effects, operational objectives and effects and tasks) to what you need in terms of capabilities. It facilitates top-down planning.

FDS is deduced to cover the full-spectrum of military operations, taking into account political guidance. Each scenario is analyzed via an adapted operational planning process.

Force Development Scenario Design (FDSD) is more a qualitative than quantitative method because usually there are not available reliable, objective and complete 'hard data' and the analyzed problem is too complex, interrelated and difficult to describe using quantified data. It applies experience, reasoning and expert opinions.

Generally, FDSD uses both exploratory and normative procedures. Exploratory approach is based on information about the past and the present, and applies heuristic approaches towards the future, so that the resulting forecast answers the questions: what could the next development be and what events or phenomena may occur in the future [5]? On the contrary, normative forecast starts with future end states, objectives, effects and tasks and returns from this future to the presence, identifying what capability, resources and technologies are necessary to attain these tasks and what constraints must be eliminated.

FDSD answers following questions:

What is the purpose of scenario?
What is the context of scenario in terms of politics, economics, social conditions and main military characteristics?

How the situation depicted in scenario may evolve?

What is the political and military end state?

What are the strategic objectives and effects we want to achieve?

What are the operational objectives and effects we want to achieve?

What are the key tasks our forces are supposed to fulfill?

Once these questions have been properly answered and it has been checked that key tasks support the overall political end-state, the mission-to task decomposition is completed. The next step of the CAMF may start.

\subsection{Capability Needs Definition}

Capability Needs Definition is critical to successful capability analysis.

Looking at the objectives, effects and tasks elaborated in FDS, planning team can begin considering what capabilities will be needed to execute tasks, generate effects and achieve objectives. To achieve the objectives, identified capabilities have to be supported by units able to deliver these capabilities. At this stage of planning, reference units (RU) are identified. RUs are units that have the ability to achieve required effects, in practice - it means the ability to fulfil key tasks identified in FDS.

One of the capability requirements would be e.g. Computer Network Operations (CNO) capability as shown in Figure 1. To have CNO capability, there has to be a force element with the ability to deliver CNO capability. This force element is described in generic terms as $\mathrm{CNO}$ unit (e.g. CNO battalion) and is considered to be a reference unit. 


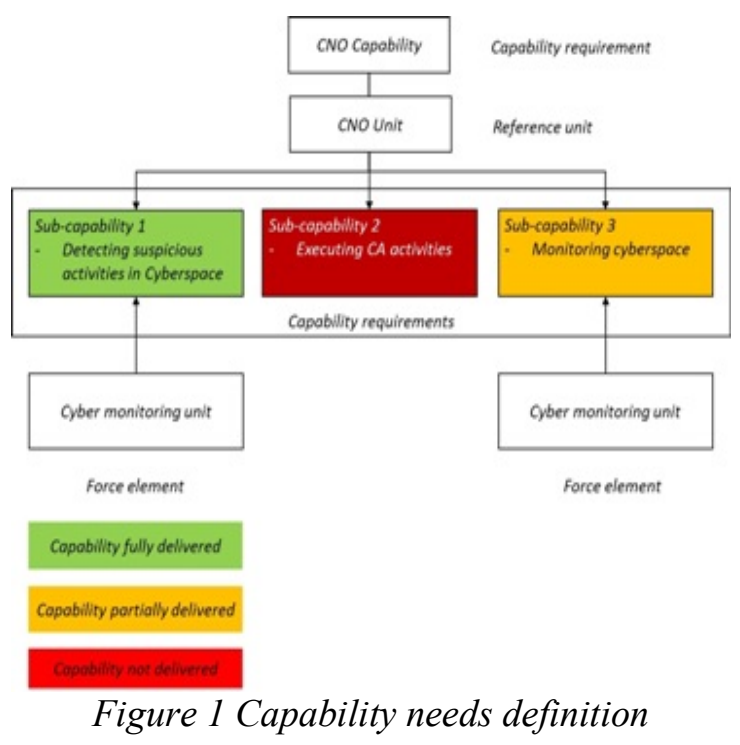

The capability requirements are drafted in generic way and are further elaborated in higher level of granularity. This forms a foundation for comparing the capability requirements to capabilities of current and planned force structure. For analytical purposes it is useful that the force structure is translated into Force Elements (FE) that might represent different groupings e.g. battalions, brigades or divisions. They may also represent main weapon systems e.g. main battle tanks, frigates or jet fighters. Capabilities of each FE are described in the same way as the capability of reference units. This construct allows for explicit and rigorous capability comparison.

CNO capability is a term at the highest level of aggregation, and it has subcapabilities at lower level of aggregation. The generic CNO Capability (Figure 1) has 3 specific sub-capabilities so that it is possible to look for the force elements that are able to deliver these specific subcapabilities. Existing Force structure has e.g. one Force Element with related capability - cyber-monitoring unit that is able to deliver fully sub-capability 1 and partially sub-capability 3 . There is no force element (existing or planned) that would be able to deliver sub-capability 2 . Using these steps, planners can identify capabilities in required level of detail.
2.4. Capability Mismatches Determination Capabilities Mismatches Determination is a process comparing capabilities of RUs and FEs. Outcomes are expressed as capability shortfall, surplus or still relevant capabilities, which should be maintained as shown in Figure 2.

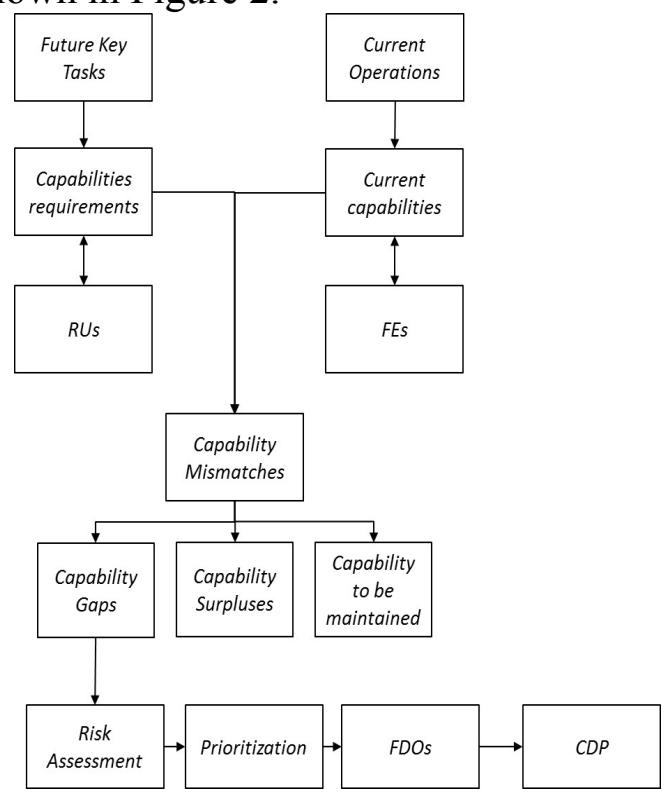

Figure 2 Capability Mismatches Determination

Capability requirements can be structured in 7 categories according to Main Capability Areas (MCA) or in an alternative structure that is considered relevant for Force development. MCA provide an aggregated description of required capabilities that FEs should achieve. When assessing the FEs' current capabilities, it is as well helpful to consider the FEs' DOTMLPFI state. It defines the force elements readiness to deliver capabilities.

Next step of MFCA is assessing operational risks related to capability gaps.

\subsection{Operational Risks Assessment}

Operational Risk Assessment (ORA) is conducted for each capability gap. That means identifying capabilities that are critical for achieving the mission objectives and end state drafted in scenarios and that have an impact on successful fulfilment of each key task.

ORA is assessed on scale 1 to 3 . 
Level of Risk 1 (LoR1) means that identified capability gap creates unacceptable risk for successful accomplishment of operation objectives. There is a high probability of mission failure and significant number of casualties and large-scale material damage will accompany that conduct of operation. Available risk mitigation measures have only limited outcome. It is an urgent requirement.

Level of Risk 2 (LoR2) means that identified capability gap is conditionally acceptable. It significantly affects achievement of operation objectives. There is a significant probability that casualties and material damage will accompany conduct of operation. Available risk mitigation measures significantly reduce associated risks to operation success. It is high priority requirement.

Level of Risk 3 (LoR3) means that identified capability gap is acceptable. It possesses only limited impact to mission accomplishment. There is low probability of casualties and material damage. Available risk mitigation measures reduce associated risks to operation success. It is low priority requirement [6].

\subsection{Prioritization of Capability Requirements}

Prioritization of capability requirements is the next step of MFCA. Based on the results of risk assessment, capability gaps are prioritized in order to minimize potential mission failure and to suffice Level of Ambitions set by the Government.

\subsection{Force Development Options}

Prioritized capability requirements provide foundation for Force Development Options (FDOs). It means that different FDOs are assessed in order to identify the most suitable solution in terms of delivery time, technical complexity and feasibility, including affordability, to mitigate capability gaps. In that regard, e.g. material and nonmaterial solutions such as training or tactics, techniques and procedures are to be considered. Solutions such as multinational cooperation or buying products of the shelf might be the correct response as well. The highest risk solutions however, are usually material ones requiring research and development. Ideally, FDOs are subject to experimentation and validation. Selected FDOs are incorporated into Capability Development Plan (CDP) and the MFCA is concluded.

\section{Conclusions}

The CAMF provides a systemic and rigorous process for determination of armed forces capabilities required for future operating environment 15 to 30 years ahead. It represents best practices already in place by several allies and NATO. Its ultimate purpose is to establish context and choice for senior leadership as they consider long-term strategic decisions related to future force development. The CAMF is a tool it is not a solution. It assists to establish a broad overview of the entire capability portfolio related to particular FDS or combination of FDSs. Additionally, it enables strategic planners to define future requirements, assess capability areas requiring investment, divestment and sustainment and identify the most suitable solution for force development.

The described methodological framework was developed and customised for a wellinformed and evidence-based decisionmaking process at the strategic level within the Czech Ministry of Defence.

\section{Acknowledgements}

Authors gratefully acknowledge both the staff of Centre of Security and Military Strategic Studies and the participants of the 31st General Staff course for their valuable insights that have been instrumental in developing the CAMF and validating the process. 


\section{References}

[1] Canadian Army Land Warfare Center, "Canada's Future Army, Volume 1: Methodology, Perspectives and Approaches", Kingston, Ontario, Canada 2015, pp. 3103.

[2] Sigurd Glaerum, Alf Christian Hennum, Analytical Support to Noewegian Long-Term Defence Planning, Czech Military Review, Brno, Czech Republic, pp. 82-91, 2016, Extraordinary volume, ISSN 1210-3292.

[3] RTO/NATO, „Handbook on Long Term Defence Planning“, St. Joseph Print Group Inc., Otawa, 2003. ISBN 92-837-1098-3.

[4] Ministry of Defence of The Czech Republic, „Defence Strategy of the Czech Republic “, Prague, Czech Republic 2012, ISBN 978-80-7278-606-0

[5] Hannah Kosow, Robert Gasner, Methods of Future and Scenario Analysis, Bonn, Germany 2008, ISSN 1860-0468.

[6] Paul R. Garvey, Analytical Methods for Risk Management, New York, USA 2009, ISBN 978-1-58488-637-2. 